\title{
Polycyclic aromatic hydrocarbons in elliptical galaxies
}

\author{
Hidehiro Kaneda ${ }^{1}$, Takashi Onaka ${ }^{2}$, Itsuki Sakon ${ }^{2}$, \\ Hiroko Matsumoto ${ }^{2}$, and Toyoaki Suzuki ${ }^{1}$ \\ ${ }^{1}$ Institute of Space and Astronautical Science (ISAS), JAXA, \\ Sagamihara, Kanagawa 229-8510, Japan \\ email: kaneda@ir.isas.jaxa.jp \\ ${ }^{2}$ Dept. of Astronomy, Graduate School of Science, The University of Tokyo, \\ Bunkyo-ku, Tokyo 113-0003, Japan
}

\begin{abstract}
We have observed 18 nearby dusty elliptical galaxies in the near- to far-infrared with Spitzer and AKARI. We have found that polycyclic aromatic hydrocarbons are present in 14 out of the 18 elliptical galaxies.
\end{abstract}

Keywords. Infrared: ISM, ISM: lines and bands, galaxies: elliptical and lenticular, cD

\section{Introduction}

Elliptical galaxies provide a unique interstellar environment, old stellar radiation fields with little UV light and interstellar space dominated by hot plasma. Far-IR emission has been detected from many elliptical galaxies (e.g., Goudfrooij \& de Jong 1995, Temi et al. 2004). The dust masses seem to be larger than those determined by the balance between replenishment by stars and sputtering destruction by plasma (Goudfrooij \& de Jong 1995), calling for additional dust-supplying sources. Recent studies show even the presence of polycyclic aromatic hydrocarbon (PAH) emission features in elliptical galaxies (e.g., Kaneda et al. 2005, 2007).

\section{Observations}

We observed 18 IRAS-detected nearby elliptical galaxies with AKARI and Spitzer; we have derived near- to far-IR 10-band images and near-IR spectra from the AKARI nearby galaxy project and mid-IR spectra from the Spitzer GO1\&3 programs.

\section{Results}

We have detected PAH emission from 14 elliptical galaxies; Figure 1 shows the Spitzer/IRS spectra of several elliptical galaxies. Most of them show unusual PAH interband strength ratios; usually strongest $7.7 \mu \mathrm{m}$ emission (e.g., Allamandola et al. 1985) is weak in contrast to prominent features at 11.3 and $17 \mu \mathrm{m}$. This may reflect the peculiar physical conditions of the ISM, i. e. dominance of large PAHs due to destruction in hot plasma, and/or dominance of neutral PAHs due to soft radiation fields from evolved stars.

The left panel in Figure 2 shows the spectral energy distribution of NGC 4589 overlaid with the Spitzer spectrum. In particular, the PAHs exhibit a prominent $17 \mu \mathrm{m}$ feature relative to the continuum emission, which makes the AKARI/IRC 16- $\mu$ m-band image an excellent tracer of large PAHs. The right panel in Figure 2 displays the AKARI 16$\mu \mathrm{m}$-band image shown after subtraction of the $7-\mu \mathrm{m}$ image with its intensity adjusted on the basis of the spectrum, which is thus likely to be the spatial distribution of PAHs. The PAH distribution appears to be different from the stellar distribution, while it has 
some resemblance to that of cool dust in the far-IR (Kaneda et al., in prep.). NGC 4589 is known to have a minor-axis dust lane as well as a complex stellar rotation field with the P.A. difference of $45^{\circ}$ between the kinematic and morphological minor axes, which suggests that the galaxy is a merger remnant (Mollenhoff \& Bender 1989). Since the distribution of the PAHs extends along the direction of the major stellar rotation, the PAHs are likely to be brought in by a merger event rather than internally produced.

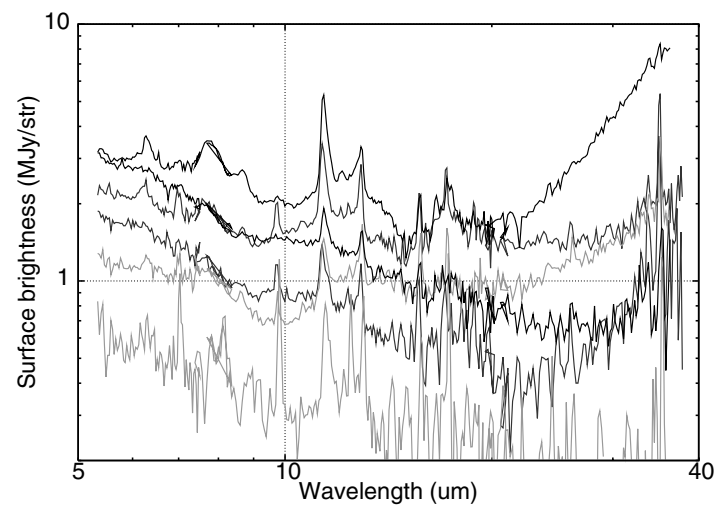

Figure 1. Spitzer/IRS spectra of elliptical galaxies obtained from our GO1\&GO3 programs. Background spectra estimated from nearby blank skies have been subtracted.
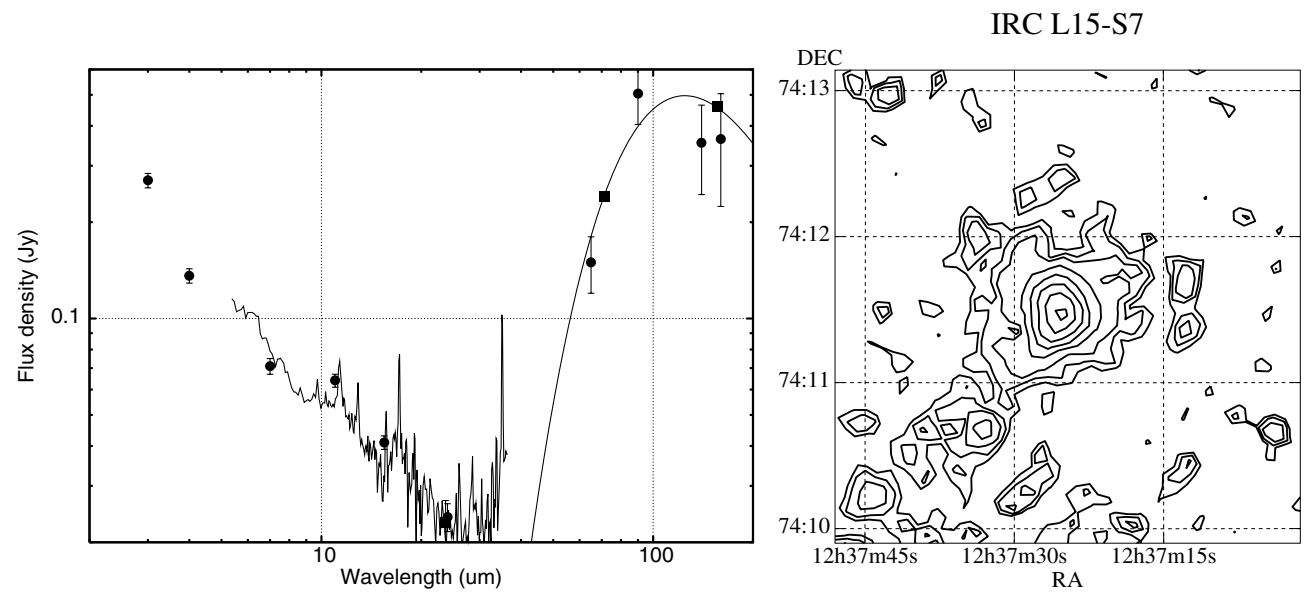

Figure 2. (Left) Spectral energy distribution of NGC4589, constructed from the AKARI (circles) and Spitzer/MIPS (boxes) photometric data, overlaid with the Spitzer/IRS spectrum. (Right) The AKARI/IRC $16-\mu \mathrm{m}$-band image shown after subtraction of the 7 - $\mu \mathrm{m}$-band image.

\section{References}

Allamandola, L. J., Tielens, A. G. G. M., \& Barker, J. R. 1985, ApJ (Letters), 290, L25

Goudfrooij, P. \& de Jong, T. 1995, A\&AA, 298, 784

Kaneda, H., et al. 2007, PASJ, 59, 107

Kaneda, H., Onaka, T., \& Sakon, I. 2005, ApJ (Letters), 632, L83

Mollenhoff, C. \& Bender, R. 1989, A\& A, 214, 61

Temi, P., Brighenti, F., Mathews, W. G., \& Bregman, J. D. 2004, ApJS, 151, 237 